\title{
THE SASKATCHEWAN BIRD ATLAS A NEW PROJECT
}

CHRISTOPHER I.G. ADAM, 2636 Argyle Street, Regina, Saskatchewan. S4S OK1 and ALAN R. SMITH, Canadian Wildlife Service, 115 Perimeter Road, Saskatoon, Saskatchewan. S7N OX4

What is a bird atlas? Simply stated a bird atlas is a collection of bird distribution maps in book form. The compilation of bird atlases is now enjoying worldwide popularity; several Canadian provinces, notably Ontario, have recently undertaken atlas projects. ${ }^{12}$ In most projects "atlasers" (mainly volunteers) determine the status of each species of bird for each of hundreds or even thousands of grid units (blocks of land). When the data for a species is transfered to a master grid the result is a map of the distribution of that species.

\section{The Saskatchewan Bird Atlas.}

The Saskatchewan Bird Atlas project is now underway. This project differs from other atlas projects in several ways. Firstly, we are initially using data that was not specifically gathered for the project. For example, we have coded most of data available from the Blue Jay and Canadian FieldNaturalist, and we are presently entering information from Canadian Wildlife Service files and the Prairie Nest Records.Scheme. Data from personal files and notebooks will also be coded in the near future. Information gathered before 1966 will be considered as historical, while information gathered from 1966 to present will be considered as current. Since the atlas has a projected completion date of 1985 , the "current" period will be 20 years long; this is the second difference as most other atlas schemes employ a 5-year period.
Thirdly, this atlas will use information gathered year-round and not just from the breeding season. However, at present, the emphasis will be placed on the accumulation of evidence of breeding. A fourth difference is the grid size, most atlas projects now under way use a 10-km square. However, since most other projects include areas of much higher population density than Saskatchewan (i.e., eastern North America and Europe) practical considerations dictate that we use a larger grid as well as the longer time period. The grid unit to be used in this project is the 1:50,000 scale mapsheet. All the grid units measure $27 \mathrm{~km}(17$ mi.) north-south, but due to the convergance of lines of longitude towards the pole they vary in width from $36 \mathrm{~km}$ (23 mi.) in the extreme south to $28 \mathrm{~km}$ (18 mi.) in the extreme north.

\section{Purpose}

The information obtained by this project will hopefully be useful in:

1) the accurate delineation of the geographic distribution of the birds of the province.

2) the identification of factors which may influence the distribution of these birds

3) the identification of any long-term and non-cyclic changes in the distribution of Saskatchewan birds and the determination of the factors that may have brought about these changes. 
4) the identification of areas of critical importance to breeding, migrating and wintering birds. Of particular interest are species with restricted ranges, or that are rare or endangered.

5) the identifcation of areas of high species diversity.

The atlas which will be the final product of this project will be a compendium of baseline data which will hopefully be useful to government agencies, environmental consultants and naturalists.

\section{How to "atlas"}

While participants may cover an unlimited number of mapsheets anywhere in the province, we would encourage you to cover one or a few mapsheets in "little known areas of the province". We will be glad to offer guidance in that regard, as well as provide you with the necessary $1: 50,000$ maps, instructions and coding forms. All that is then required is that you spend at least a few hours on a mapsheet and record your observations for each species by entering the appropriate status code (see below) on the atlas coding form. A species' status code can be upgraded on subsequent visits in the same or later years.

There are qualifications to several of these codes. For example, owls tend to nest earlier than other species, so breeding evidence for owls may be sought outside the "normal" breeding season; other species such as gulls may forage in 'apparently suitable nesting habitat' without actually nesting. Complete details are available in the first atlas newsletter. ${ }^{3}$ Also note these changes in status codes as presented in the newsletter: codes B8 and B9 (probable breeding) are upgraded to BB8 and BB9 (confirmed breeding).

\section{Participation}

In most atlas projects volunteer work plays an important part since it is the amateur birders who collect most of the data. Anyone with some experience in identifying birds may participate in this project. Even if you do not wish to participate in the field work, your past records will be of great value to us. If you do not wish to code your past records, we will code them if you make your records available to us.

It is hoped that this project is structured in such a way that it can be fun and yet still yield information of scientific value. Parties may wish to get together and blitz a mapsheet; this may be an appropriate activity for the upcoming SNHS summer meet.

Anyone wishing to participate in the project is asked to contact:

Alan R Smith Overall Coordinator and Coordinator for south of $54^{\circ}$, Canadian Wildlife Service, 115 Perimeter Road, Saskatoon, Saskatchewan. S7N 0X4 (Tel. 665-4091 or 665-4087) or Christopher I.G. Adam Coordinator for north of $54^{\circ}, 2636$ Argyle Street, Regina, Saskatchewan. S4S 0K1 (Tel. 584-9564)

\section{Progress to Date}

We, along with our assistant Mary Gilliland, have made over 25,000 entries into the atlas. This is $12.5 \%$ of the estimated 200,000 records that would be necessary to complete the atlas. Several preliminary maps have been produced and one of them is included herein (Figure 1). This map of Burrowing Owl distribution is based primarily on the results of a questionnaire conducted by J.A. Wedgewood for the Committee on the Status of Endangered Wildlife in Canada (COSEWIC). ${ }^{4}$ Recent fieldwork, however, has upgraded the status of this speices on about 10 mapsheets. 


\section{Saskatchewan Bird Atlas Status Codes}

The following is an abridged list of the status codes to be used in this project:

W - Winter Visitant - Recorded between 15 December and 29 February

S - Spring Migrant - Recorded between 1 March and 31 May

F - Fall Migrant - Recorded between 1 August and 14 December

$\checkmark$-Summer - Recorded between 1 June and 31 July without evidence of Visitant breeding

$\mathrm{P} \quad-$ Possible $-P-1 . \quad$ Observed in suitable nesting habitat during the breeding Breeding season (generally from 1 June to 31 July)

- P-2. $\quad$ Singing male(s) present or breeding song heard during the breeding season in suitable nesting habitat.

B - Probable - B-3. Pair observed during the breeding season in suitable Breeding nesting habitat

- B-4. Courtship and display noted during the breeding season in suitable nesting habitat

- B-5. Permanent territory presumed through the registration of territorial behavior on 2 days at least a week or more apart at the same place

$-B-6 . \quad$ Visiting probable nest-site

-B-7. Agitated behavior or anxiety calls from adults

BB - Confirmed - BB-8. Brood patch on adult examined in the hand Breeding

-BB-9. Nest-building or excavation of nest-hole

$-B B-10$. Distraction display or injury-feigning

$-B B-11$. Used nest or eggshells found

-BB-12. Recently-fledged young (nidicolous species) or downy young (nidifugous species)

- BB-13. Adults entering or leaving nest-site in circumstances indicating occupied nest (including high nests or nestholes, the contents of which cannot be seen) or adult seen incubating

- BB-14. Adult seen carrying faecal sac or food for young

$-B B-15$. Nest containing eggs; includes cowbird eggs

$-B B-16$. Nest containing young; includes cowbird young 
- Former breeding range
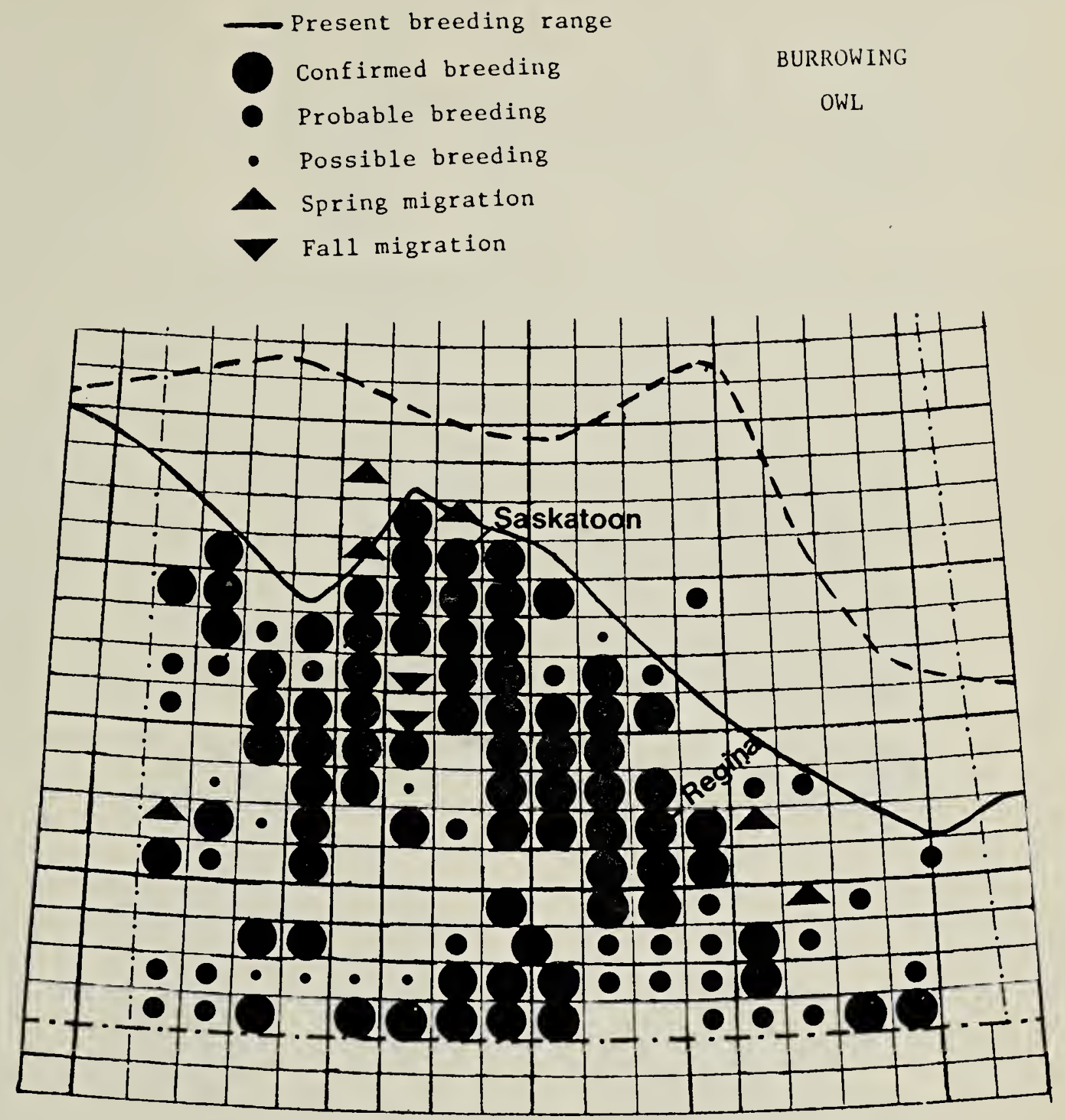

Figure 1.The distribution of the Burrowing Owl in Saskatchewan. The dash-dot line indicates the provincial boundary. Rectangles are 1:50,000 mapsheets.

Volunteers have already made a significant contribution to this project. Brenda Dale, Brian Johns, Chris Harris, Dale Hjertaas, Bohdan Pylypec and Stan Shadick have collectively contributed over 1000 records. Our thanks to these people for their efforts.

ITHE ALBERTA ORNITHOLOGICAL RECORDS COMMITTEE. 1982. The Alberta Breeding Bird Atlas: a guide to participants. 1982: pilot project year. Federation of Alberta Naturalists.
${ }^{2}$ CADMAN, M., editor. Ontario Breeding Bird Atlas Newsletters. Nos. 1,2,3 and 4. Ontario Breeding Bird Atlas.

${ }^{3}$ SMITH, A., editor. 1983. Saskatchewan Bird Atlas Newsletter. Vol. 1(1). March 1983.

${ }^{4}$ WEDGWOOD, J.A. 1979. Status of the Burrowing Owl in Canada in Status Reports of Endangered Wildlife in Canada. Committee on the Status of Endangered Wildlife in Canada, Ottawa. 